ARTICLE

Received 16 Jun 2014 | Accepted 12 Jan 2015 | Published 27 Feb 2015

DOI: $10.1038 /$ ncomms 7274

\title{
AtNIGT1/HRS1 integrates nitrate and phosphate signals at the Arabidopsis root tip
}

\author{
Anna Medici ${ }^{1}$, Amy Marshall-Colon ${ }^{2, \dagger}$, Elsa Ronzier ${ }^{1} \dagger$, Wojciech Szponarski ${ }^{1}$, Rongchen Wang ${ }^{3}$, Alain Gojon ${ }^{1}$,
} Nigel M. Crawford ${ }^{3}$, Sandrine Ruffel ${ }^{1}$, Gloria M. Coruzzi ${ }^{2} \&$ Gabriel Krouk $^{1}$

Nitrogen and phosphorus are among the most widely used fertilizers worldwide. Nitrate $\left(\mathrm{NO}_{3}^{-}\right)$and phosphate $\left(\mathrm{PO}_{4}{ }^{3-}\right)$ are also signalling molecules whose respective transduction pathways are being intensively studied. However, plants are continuously challenged with combined nutritional deficiencies, yet very little is known about how these signalling pathways are integrated. Here we report the identification of a highly $\mathrm{NO}_{3}^{-}$-inducible NRT1.1-controlled GARP transcription factor, HRS1, document its genome-wide transcriptional targets, and validate its cis-regulatory elements. We demonstrate that this transcription factor and a close homologue repress the primary root growth in response to $\mathrm{P}$ deficiency conditions, but only when $\mathrm{NO}_{3}^{-}$is present. This system defines a molecular logic gate integrating $\mathrm{P}$ and $\mathrm{N}$ signals. We propose that $\mathrm{NO}_{3}^{-}$and $\mathrm{P}$ signalling converge via double transcriptional and post-transcriptional control of the same protein, HRS1.

\footnotetext{
${ }^{1}$ Biochimie et Physiologie Moléculaire des Plantes, Institut Claude Grignon, UMR5004 CNRS/INRA/Supagro-M/UM2, Place Viala, F-34060 Montpellier cedex 2, France. ${ }^{2}$ Center for Genomics and Systems Biology, Department of Biology, New York University, New York, New York 10003, USA. ${ }^{3}$ Section of Cell and Developmental Biology, Division of Biological Sciences, University of California at San Diego, La Jolla, California 92093-0116, USA. †Present addresses: Department of Plant Biology, University of Illinois, 1201W Gregory Drive, 193 ERML MC-051, Urbana, Illinois, USA (A.M.-C.); Department of Medicine, Aab Cardiovascular Research Institute, University of Rochester School of Medicine and Dentistry, Rochester, New York, New York 14642, USA (E.R.).

Correspondence and requests for materials should be addressed to G.K. (email: gkrouk@gmail.com).
} 
A s sessile organisms, plants have evolved a myriad of adaptive mechanisms to cope with nutritional limitations in their environment. In particular, plants adapt their root development differently according to nutritional cues ${ }^{1}$. Nitrate $\left(\mathrm{NO}_{3}^{-}\right)$and phosphate $\left(\mathrm{PO}_{4}{ }^{3-}\right)$, two major phytomacronutrients, are also well known signalling molecules shaping root development through partly defined molecular pathways ${ }^{2-6}$. For the inorganic phosphate (Pi) response, $\operatorname{prd} d^{7}, \operatorname{lpr} 1, \operatorname{lpr} 2$ (ref. 8), pdr2 (refs 9,10), phr1 (refs 11,12) and siz1 (ref. 13) mutations have been found to affect the primary root growth. Concerning $\mathrm{NO}_{3}^{-}$, its effect is more related to lateral root growth, with the implication of several molecular actors (thoroughly reviewed in refs 4,14), and has been shown to counteract the effect of glutamate on the primary root growth ${ }^{15}$.

Despite this knowledge, a mechanism by which a plant integrates the presence or the absence of combinations of such key nutritional molecules is still largely unknown. Recently, the NLA (nitrogen limitation adaptation) and $\mathrm{PHO} 2$ proteins (two ubiquitin ligases) were found to control $\mathrm{PO}_{4}^{3-}$ transporter trafficking (PHT1 family), which results in a nitrogen-dependent $\mathrm{PO}_{4}^{3-}$ accumulation in leaves ${ }^{16-19}$. Despite these first hints into the molecular connections between $\mathrm{P}$ and $\mathrm{N}$ nutrition, nothing has so far been revealed concerning the mechanisms by which $\mathrm{NO}_{3}^{-}$affects $\mathrm{PHO} 2$ or NLA activities.

The root tip is known to be at the forefront of Pi sensing 8 . It is also the territory of expression of the recently identified nitrate sensor NRT1.1 (refs 20,21). Thus, it represents the perfect place for such nutritional signalling interactions. The present work reports such missing mechanism. Here we show that HRS1 and $\mathrm{HHO} 1$ are two early $\mathrm{NO}_{3}^{-}$-regulated transcription factors (TFs). We document genome-wide HRS1 direct targets and demonstrate that hrs1; hhol double mutant is involved in the primary root growth repression in response to a combination of $\mathrm{N}$ and $\mathrm{P}$ signals. We report a potential mechanism to explain this phenotype as HRS1 is under a dual transcriptional and posttranscriptional control. Finally, an in planta genome-wide investigation provides potential signalling pathways under HRS1 and HHO1 influence.

\section{Results}

Identification of two $\mathrm{NO}_{3}^{-}$-regulated TFs. A set of early nitrateregulated gene clusters have been previously identified in a genome-wide investigation ${ }^{22}$. Among these, At1g13300 was one of the most rapidly and strongly upregulated TFs (with a significant response recorded within $6 \mathrm{~min}$ after treatment). The early and marked induction of At 1 g13300 and several genes involved in the nitrate uptake and assimilation have been confirmed in an independent set of experiments (Fig. 1). In roots, $\mathrm{NO}_{3}^{-}$provision triggers the induction of the two sentinel gene transcripts (NIR and NRT2.1) after 10 and $30 \mathrm{~min}$ respectively. This validates the conditions of the $\mathrm{N}$ treatment (Fig. 1a). As predicted by the previous experiments, At1g13300 accumulation was rapidly and strongly upregulated (10-fold within $10 \mathrm{~min}$ ) compared with the $\mathrm{KCl}$ mock treatment (Fig. 1a). These observations are consistent with the previous reports corresponding to genome-wide or specific reverse transcription quantitative (qPCR) investigations ${ }^{6,23-25}$.

The At1g13300 gene encodes a myb-related TF belonging to the GARP (GOLDEN2, ARR-B, Psr1) family; a homologue to NIGT1 identified in rice ${ }^{26}$. The GOLDEN2-like subgroup is composed of 40 protein sequences (AGRIS, http://arabidopsis. med.ohio-state.edu/). It encompasses proteins involved in various processes ranging from the control of chloroplast and leaf development $\left(\mathrm{GLKs}^{27}\right.$ and KANADIs ${ }^{28}$ ) to nutritional reprogramming in plants (PHR1 and PHL1 (ref. 12)). A phylogenetic tree has been built out of the 40 G2-like protein sequences (Supplementary Fig. 1, see Methods). It shows that At1g13300 belongs to a small group of seven proteins sharing high sequence similarity to each other, but quite well separated from the rest of the tree (Fig. 1c; Supplementary Fig. 1). This subgroup within the larger GARP family has already been reported on its own ${ }^{29}$. We thus kept the proposed nomenclature and extended the relationships between the other members of the GARP family. Atlg13300 is named HRS1 (hypersensitive to low Pi-elicited primary root shortening 1$)^{29}$. HRS1 is a paralogue with its closest neighbour, At3g25790, which has been named HHO1 (HRS1 homologue 1$)^{29}$. It is noteworthy that these two close homologues, HRS1 and HHO1, have recently been identified among the 2,594 gene pairs that were defined in an effort to isolate redundant duplicated genes ${ }^{30}$. Interestingly, $\mathrm{HHOl}$ showed a similar pattern of expression to HRS1 with a strong upregulation ( $>50$-fold) within the first $20 \mathrm{~min}$ of nitrate treatment (Fig. 1a), both being mainly expressed in roots (Fig. 1d). Furthermore, HRS1 and HHO1 messenger RNA (mRNA) levels in roots are dependent on nitrate concentration in the media. Wild Type seedlings constantly grown for 14 days on media containing increasing $\mathrm{NO}_{3}^{-}$concentrations presented a gradual transcriptional response of HRS1 and HHO1 (Fig. 1b). Interestingly, despite the fact that both genes are upregulated by $\mathrm{NO}_{3}^{-}$provision, the increasing $\mathrm{KNO}_{3}$ concentration has opposite effects on their steady-state expression levels: HRS1 is positively regulated and HHO1 is negatively affected (Fig. 1b). In conclusion, HRS1 and $\mathrm{HHO} 1$ are two root-specific, $\mathrm{NO}_{3}^{-}$controlled TFs (found to be responsive in the nitrate reductasenull mutant in previous genome-wide investigations ${ }^{31}$ ). A meta-analysis ${ }^{6}$ also demonstrated that HRS1 and HHO1 are under the control of NRT1.1 protein activity. Indeed, their nitrate induction is strongly and robustly affected in chll mutants (defective in the NRT1.1 gene) in two independent transcriptomic data sets issued from two independent laboratories ${ }^{25,32}$. We also performed analysis in our conditions and indeed recorded defects in nitrate responsiveness in an independent chl1 deletion allele of NRT1.1 (chl1-12; Fig. 1e). In the chl1-12 mutant, the 24-fold induction (after $30 \mathrm{~min}$ of $\mathrm{NO}_{3}^{-}$treatment) of HRS1 expression is totally abolished and the HHO1 strong induction ( $\sim 100$-fold) is reduced to a third. Finally, HRS1 and HHO1 are also under the influence of NLP genes (NIN-like TFs) implicated in $\mathrm{N}$-signalling ${ }^{33}$, and are found to be bound (assayed by chromatin immunoprecipitation sequencing) by NLP7 (ref. 34).

Collectively, these results demonstrate that the TF paralogues, HRS1 and $\mathrm{HHO} 1$ are each strongly $\mathrm{NO}_{3}^{-}$controlled (for example, are among the most, if not the most, robustly $\mathrm{NO}_{3}^{-}$regulated genes in many data sets ${ }^{35}$ ) and positioned downstream of the early regulators NRT1.1/CHL1 and NLP7-6 activity.

Identification of HRS1 direct targets. At the outset of this study nothing was known about the molecular mechanism downstream of At1g13300 (HRS1). We thus decided to investigate the genome-wide effect of this TF using a transient assay system for TF perturbation that can uncover direct targets only ${ }^{36}$. Indeed, we have shown for other TFs (ABI3 and bZIP1) that using this system to uncover the direct regulated targets of a TF can give very important clues about its functional role in planta ${ }^{36,37}$. We thus speculated that we could retrieve the functional in planta activity of Atlg13300 by studying the set of its direct targets.

To search for such genes that are directly regulated by Atlg13300, we used the TARGET (Transient Assay Reporting Genome-wide Effects of Transcription factors) approach described by Bargmann et al. ${ }^{36}$ Briefly, protoplasts are isolated from roots of 10-day-old Arabidopsis seedlings and transformed with the plasmid pBeaconRFP_GR-HRS1, expressing a 

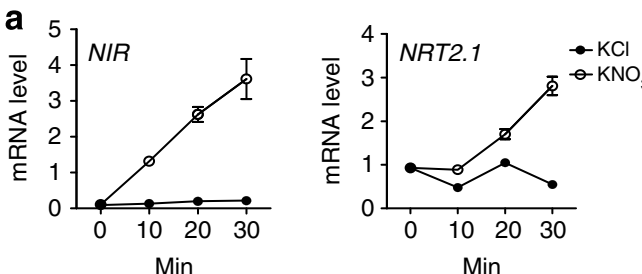

b
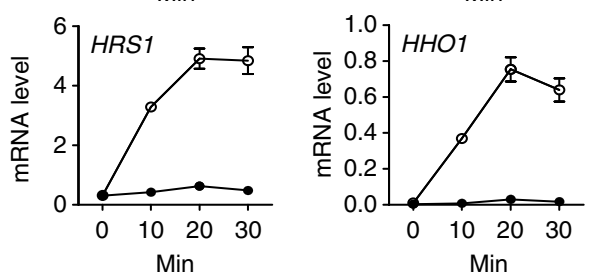

C
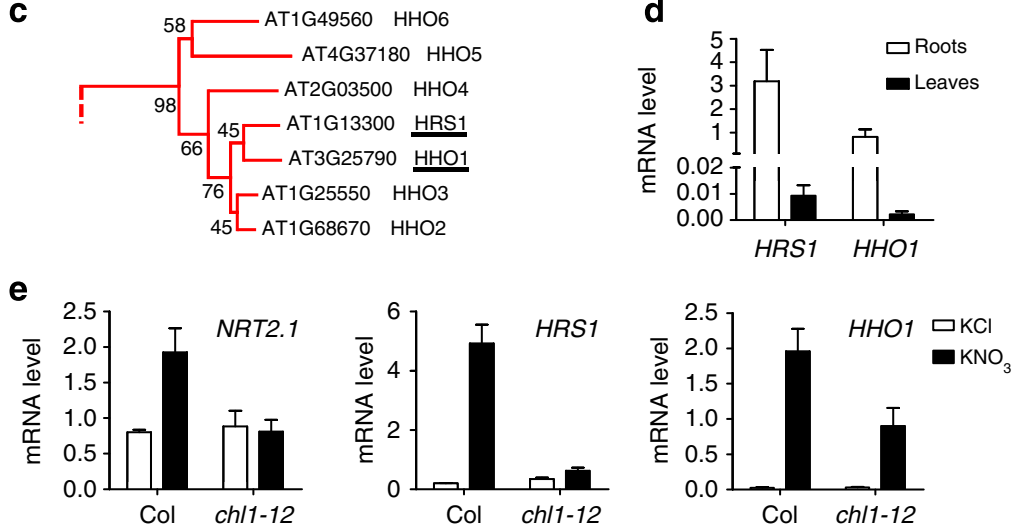

$\mathbf{f}$

551 regulated probes

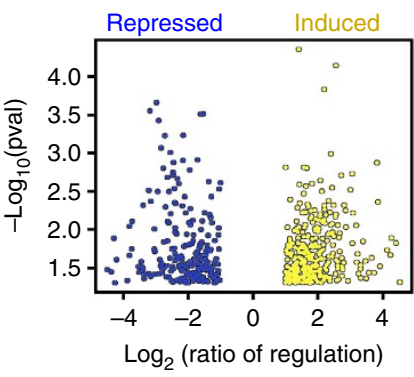

\section{g}
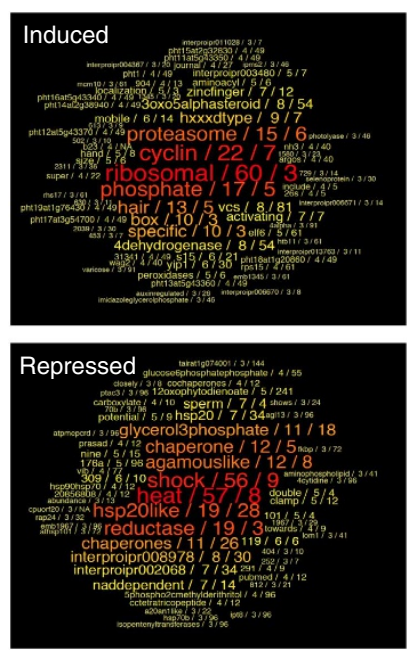

Figure 1 | HRS1 and $\mathrm{HHO1}$ are two Arabidopsis transcription factors highly induced by $\mathrm{NO}_{3}^{-}$downstream the nitrate sensor activity. Identification of HRS1 direct targets. (a) Dynamic nitrate response of mRNA for NIR, NRT2.1 (NO3-- responsive sentinels), HRS1 (At1g13300) and HHO1 (At3g25790) in roots of 14-day-old seedlings treated with $1 \mathrm{mM} \mathrm{KNO}_{3}$ or $1 \mathrm{mM} \mathrm{KCl}$ (as mock treatment), values are means \pm s.e.m. $(n=4)$. (b) Steady-state level of $\mathrm{HRS1}$ and $\mathrm{HHO} 1$ mRNA in roots of plants grown for 14 days on solid media containing $0.5 \mathrm{mM} \mathrm{KH}_{2} \mathrm{PO}_{4}$ and different $\mathrm{KNO}_{3}$ concentrations, values are means \pm s.e.m. $(n=4)$. (c) A subfamily of the G2-like transcription factors phylogenetic tree built by ClustalW alignment and maximum likelihood method, values are bootstraps based on 500 replicates (see Supplementary Fig. 1). (d) HRS1 and HHO1 mRNA levels in roots and leaves of WT plants grown for 14 days on basal MS media containing $0.5 \mathrm{mM} \mathrm{KH}_{2} \mathrm{PO}_{4}$ and $2.5 \mathrm{mM} \mathrm{KNO}_{3}$, values are means \pm s.e.m. $(n=3)$. (e) HRS1 and $\mathrm{HHO1}$ nitrate induction is affected in roots of chl1-12 mutant mutated in NRT1.1. WT and chl1-12 14-day-old seedlings were treated for 30 min with $1 \mathrm{mM} \mathrm{KNO}$ or $1 \mathrm{mM} \mathrm{KCl}$ (as mock treatment). All transcript levels were quantified by reverse transcription qPCR and normalized to two housekeeping genes (ACT and CLA), values are means \pm s.e.m. $(n=4)$. (f) Volcano plot of HRS1 direct regulated targets identified by TARGET (transient assay reporting genome-wide effects of transcription factors) procedure ${ }^{36}$. (g) Semantic gene enrichment analysis results showing overrepresented terms in the list of the HRS1 direct up- and downregulated genes.

translational fusion (N-ter) between HRS1 and the rat glucocorticoid receptor (GR) under the control of the pCaMV35S promoter. Protoplasts are treated with (i) dexamethasone (DEX; triggers GR-TF fusion entrance in the nucleus) and (ii) cycloheximide (CHX; translation inhibitor that prevents activation of indirect targets) ${ }^{36}$. Because of the possibility that HRS1 acts as a part of $\mathrm{a} \mathrm{NO}_{3}^{-}$-regulated protein complex (as it is itself $\mathrm{NO}_{3}^{-}$regulated, see above), we kept $\mathrm{NO}_{3}^{-}$ present during the whole TARGET procedure. After fluorescentactivated cell sorting selection (based on red fluorescent protein signal provided by an independent cassette in the plasmid) of transformed protoplasts, total RNA was isolated from GR-HRS1 protoplasts and used for the transcriptomic analysis. The statistical analysis (see Methods) of transcriptome results identified 551 gene probes whose expression was affected by GR-HRS1 nuclear import. The corresponding genes were classified as up- and downregulated HRS1 direct target genes (Fig. 1f; Supplementary Data 1).

To search for the functions that are globally affected by HRS1 activity, we performed two kinds of analysis. First, we determined over-represented gene ontology (GO) categories using the VirtualPlant and agriGO platforms ${ }^{38,39}$. Second, we developed our own algorithm that search for over-represented terms in a set of TAIR v10 gene description (see Methods). Both approaches yielded very similar conclusions: At1g13300 (HRS1) collectively induces genes related to 'phosphate' and 'cell division' (including the terms meristematic activity, cyclin and ribosomal proteins). On the other hand, in the list of HRS1 downregulated genes, the most frequent terms were 'heat' 'shock' and the most represented functions were linked to response to stresses (Fig. 1g; Supplementary Data 1; Supplementary Fig. 6). This genome-wide investigation of the At1g13300 HRS1 TF direct targets brought us to be interested in the interaction between $\mathrm{NO}_{3}^{-}$signalling (that controls transcriptional activation of this gene) and $\mathrm{P}$ nutrition that seems to be one of the functions under its influence ${ }^{29}$. Taken together with the fact that HRS1 belongs to the same TF family as the very well characterized PHR1 gene ${ }^{11,12}$, known to be central in the control of $\mathrm{P}$ starvation response, we decided to employ a reverse genetic approach to understand the role of HRS1 in the control of $\mathrm{NO}_{3}^{-}$and $\mathrm{Pi}$ signalling interactions.

HRS1 and HHO1 repress primary root growth. The hrs 1-1 mutant was obtained from ABRC seeds stock center, and the 
T-DNA insertion and the absence of HRS1 full-length transcripts were confirmed (Supplementary Fig. 2). First, we tested the growth of the hrs 1-1 mutant on different media containing $+\mathrm{P}$ $\left(0.5 \mathrm{mM} \mathrm{KH}_{2} \mathrm{PO}_{4}\right)$ or $-\mathrm{P}$ combined with different $\mathrm{NO}_{3}^{-}$concentrations $(0,0.05,0.5,1$ and $2.5 \mathrm{mM})$. The results did not lead to any reliable/robust phenotype (Supplementary Fig. 3a), as previously reported ${ }^{29}$. We thus hypothesized that this could be due to functional redundancy of the two $\mathrm{NO}_{3}^{-}$-induced close relatives HRS1 and HHO1 (Fig. 1c). We thus tested the double mutant hrs1-1;hho1-1 (see molecular characterization in Supplementary Fig. 2) on the same $\mathrm{P} / \mathrm{N}$ varying conditions. As previously shown ${ }^{8}$, the primary root growth of WT plants was impaired on $-\mathrm{P}$ conditions. Interestingly, however, the primary root growth of the double hrs1; hhol mutant was strongly insensitive to the $-\mathrm{P}$ conditions (Fig. 2a,b). This insensitivity is also manifested on transfer experiments (Supplementary Fig. 3b,c). The fact that the hsrl;hhol double mutant is resistant to $-\mathrm{P}$ conditions complements previous observations reporting that the overexpression of HRS1 indeed confers hypersensitivity to low $\mathrm{P}$ conditions ${ }^{29}$. More importantly, $\mathrm{NO}_{3}^{-}$ is required for the manifestation of the hrs1; hhol double mutant phenotype. Indeed, the phenotype of the hrsl;hhol double mutant is lost in plants grown on $-\mathrm{N} /-\mathrm{P}$ conditions. More precisely, the presence of at least $0.05 \mathrm{mM} \mathrm{NO}_{3}^{-}$in the media was necessary for the appearance of the root phenotype in response to Pi-depleted conditions (Fig. 2c). These results suggest that the HRS1 and HHO1 proteins act as repressors of primary root development specifically when $\mathrm{PO}_{4}^{3-}$ is absent and $\mathrm{NO}_{3}^{-}$is present in the media. It is thus tempting to propose that the well-known primary root growth repression in response to low $\mathrm{P}$ conditions is an active process that is influenced by (i) $\mathrm{NO}_{3}^{-}$and (ii) the $\mathrm{NO}_{3}^{-}$-regulated TFs HRS1 and HHO1.

HRS1 and HHO1 are expressed in elongating root cell nuclei. To further investigate the in planta roles of the HRS1 and HHO1 proteins, we constructed native promoter gene green fluorescent protein (GFP) lines for these two TFs. Epifluorescence and confocal imaging of transgenic $p H R S 1: H R S 1: G F P$ and $p H H O 1: H-$ HO1:GFP plants indicate that the two proteins are expressed and localized in the nucleus of epidermal and cortex cells in elongating roots (Fig. 2d; Supplementary Fig. 4). HRS1 and HHO1 expression is found in the transition domain of the root apical meristem and in the elongation zone (Fig. 2d). The expression of HRS1 was also visible in root hair cells (Supplementary Fig. 4). The localization of these two proteins supports the idea that a
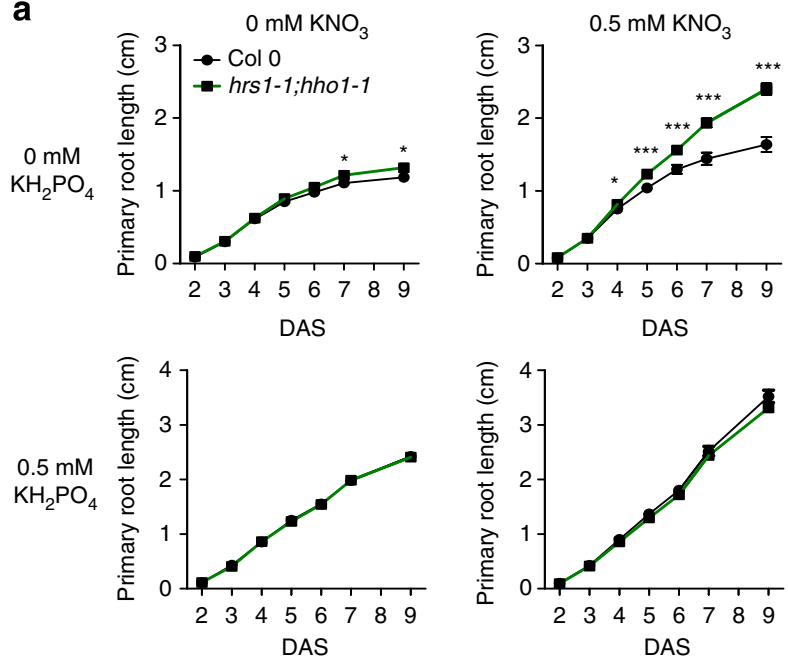

b

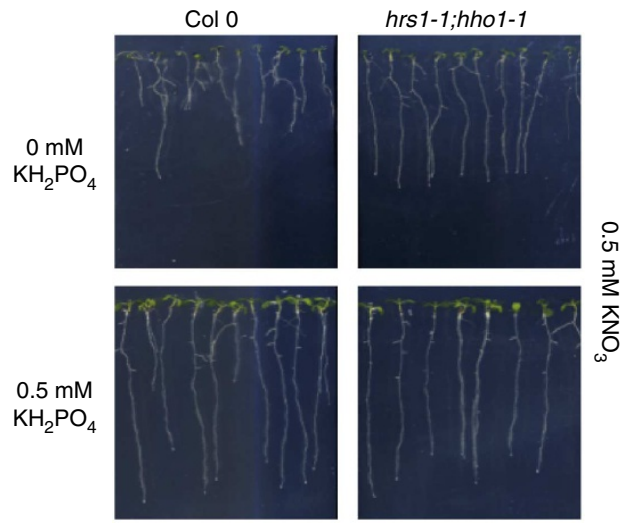

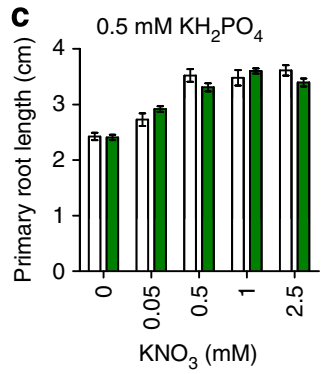

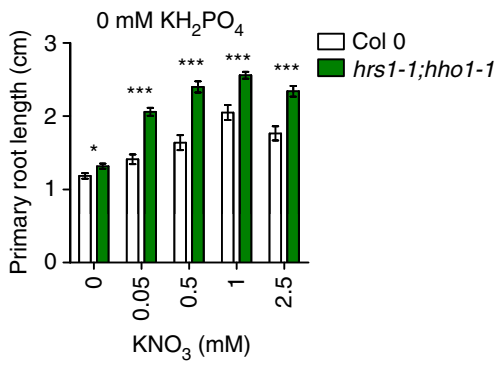

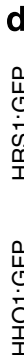
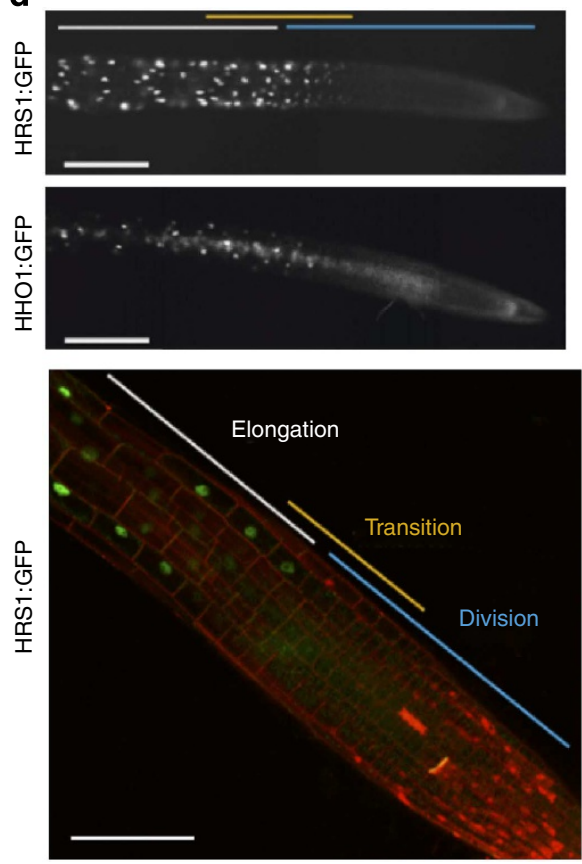

Figure 2 | HRS1 and HHO1 are two nuclear factors expressed in meristematic elongating cells repressing root growth in response to combination of $\mathbf{P}$ and $\mathbf{N}$ availability. (a) Primary root growth measurement over time of Col and hrs1-1; hho1-1 seedlings grown on $\mathrm{P} / \mathrm{N}$ combinations. In $+\mathrm{P} /-\mathrm{N}$ conditions, the WT and mutant slopes overlap. (b) Nine-day-old seedlings grown on $0.5 \mathrm{mM}$ of $\mathrm{NO}_{3}^{-}$and $\mathrm{PO}_{4}^{3-}$ under different conditions (0-0.5 mM added). (c) Effect of different nitrate concentrations in the media on the primary root growth of hrs 1-7; hho1-1 9 day-old seedlings. Values represent the means \pm s.e.m. $(n=30)$. Asterisks indicate significant differences from WT plants $\left({ }^{\star} P<0.05 ;{ }^{\star \star \star} P<0.001\right.$; Student's $t$-test). (d) Epifluorescent and confocal imaging of pHRS1:HRS1:GFP and pHHO1:HHO1:GFP expressing plants (scale bars, $200 \mu \mathrm{m}$ ). 
HRS1 and HHO1 are nuclear-localized TFs involved in the control of the root elongation.

HRS1 binds to two different cis-regulatory elements. To identify cis-regulatory elements (CREs) directly targeted by HRS1 protein, we searched for conserved motifs in the promoters of the most strongly up- and down-regulated target genes. Thus, we examined the $500 \mathrm{bp}$ promoter sequences upstream the transcription start site for the 120 top induced and repressed genes (based on fold regulation upon DEX treatment) from the TARGET transcriptome analysis (presented in Fig. 1f). Five strongly significant motifs were retrieved using the MEME algorithm ${ }^{40}$. The two cis-motifs, which were over-represented in the promoters of genes upregulated by HRS1, display the consensus sequences AGANNNAAA and AAACNNAACC. By contrast, the HRS1 downregulated genes share three over represented motifs: AANNAGA, TGGGC and GAGA (Fig. 3a). Among these last three cis-motifs, the TGGGC $(\mathrm{C} / \mathrm{T})$ motif was already known as the core-binding sequence of the CRE for TCP (teosinte branched1, cycloidea, PCF) $\mathrm{TFs}^{41}$. Inspired by the consensus sequences above, we defined five motifs (M1-M5; Supplementary Fig. 5c; Supplementary Table 1) that were tested in vitro for direct binding by HRS1 in Electophoretic Mobility Shift Assays (EMSAs). The HRS1 recombinant protein produced a shift for motifs \#1, 2, 3 and 5 . The shifts were completely reverted by the competition of a 200-fold molar excess of the same unlabelled probe (Supplementary Fig. 5d). No band shift was detected for motif \#4, which corresponds to the TCP-binding site. This means that TCP TFs may be partners of HRS1 mediating its repressive transcriptional activity. Motifs \#1, 2 and 3 in the HRS1-induced genes share the core sequence AGA and the motif \#5 the core AACC (already known as part of the GLK1/2-binding site ${ }^{27}$ ). This is in accordance with the recent finding that a particular TF can bind to distinct cis-elements ${ }^{42}$. The specificity of HRS1 binding to the selected DNA probes was confirmed by EMSA analysis, using unlabelled probes mutated on the above described core sequences (AGA and AACC). Ten-, 25-, and 50-fold molar excess of unlabelled mutant probes could not compete with HRS1 binding to motifs \#1, 3 and 5, thus confirming that HRS1 binding is maintained by AGA and AACC core sequences (Supplementary Fig. 5e).

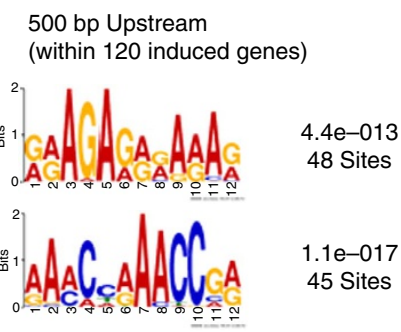

500 bp Upstream

(within 120 repressed genes)

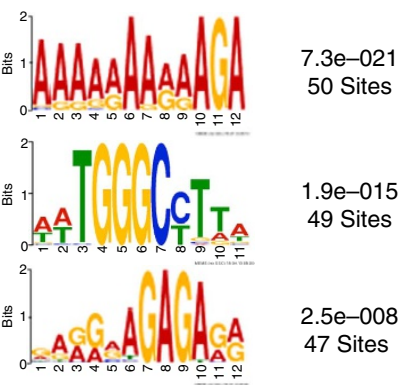

b

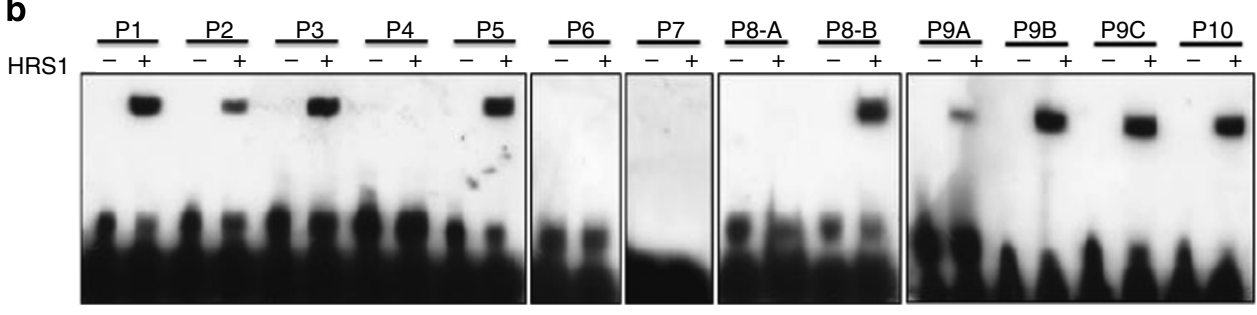

C

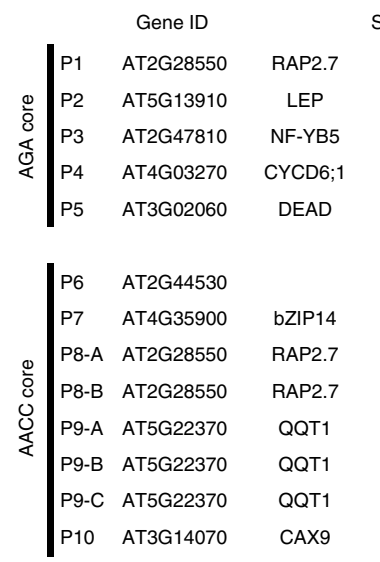

Strand Start $P$-value

$4454.16 \mathrm{e}-06$

$4096.43 \mathrm{e}-06$

$321 \quad 2.91 \mathrm{e}-06$

$432 \quad 4.16 \mathrm{e}-06$

$489 \quad 1.45 \mathrm{e}-07$

$367 \quad 1.41 \mathrm{e}-05$

$487 \quad 6.06 \mathrm{e}-06$

$340 \quad 1.21 \mathrm{e}-05$

$84 \quad 2.24 \mathrm{e}-06$

$352 \quad 1.14 \mathrm{e}-07$

$415 \quad 1.34 \mathrm{e}-07$

$330 \quad 1.910-06$

$\begin{array}{ll}330 & 1.91 \mathrm{e}-06 \\ 440 & 5.35 \mathrm{e}-06\end{array}$
Site

tTAtAgttac agagagagaAaA acAactttat *

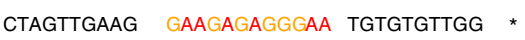

AAGAAAATTG agAGAGAGAGAA ATtAGCtAGA *

GTTAGAAGAT GAAGAAGAGAG AGAGGGACAG

AGGGGATAAT GAAGAGAGAGAG AGAGAGAGTG *

AGACCTTCCG AAAATAAACCGA ATTTCATCGG

TGGAGTCTTG GACCCAACCCCA TC

AAAAAAAAAA AAAAAAAACCGG AAACTTACTT

GTCACCCCAA AACCCAAACCAA ACGACGTGAA *

GGAGTATCCG AACCCAAACCGA AAAGTGAATT *

TGCTATACTC GAACCGAACCGG ATAATCGAAA *

ATCCGAAAAC AAACCGACCCGA AATTCACTTT *

ATAAACCCCA AAACCTAACCGA ACCAGAAAGG *

Figure 3 | Identification of cis-regulatory elements and binding of HRS1 to the promoters of 'meristem-related' genes. (a) Weight matrix representation of the motifs retrieved by the MEME algorithm analysis from the 500 bp sequences upstream the transcription start sites of the top 120 direct up- and downregulated HRS1-target genes. (b) EMSA analysis on 30-bp promoter fragment from HRS1 directly regulated genes (from TARGET analysis); biotin-labelled DNA probes (20 fmol), HRS1-GST protein (50 $\mathrm{ng}$ ). (c) List of the promoter fragments sequences used for EMSA analysis in $\mathbf{a}$. For each promoter the gene ID, the common name and the strand position is indicated. 
To obtain functional explanations of the root developmental phenotype described (Fig. 2a-c), we tested whether HRS1 was also able to bind native CREs found in promoters of genes categorized as 'meristem-related' genes. For that we used 30-bp promoter fragments as EMSA probes. HRS1 produced a significant shift for 9 of 13 promoter fragments tested (Fig. 3b,c). Promoters have been selected as they are; (i) induced by HRS1 in the TARGET system (Fig. 1f); and (ii) they are classified by GO as being involved in meristematic activity. To summarize, HRS1 is shown to be able to bind the promoter sequences of RAP2-7 (related to AP2.7; AT2G28550), LEP (leafy petiole; AT5G13910), the NF-YB5 (nuclear factor Y, subunit B5; $A T 2 G 47810) \mathrm{TF}$, the helicase DEAD (AT3G02060), the DNAbinding protein QQT1 (quatre quart 1; AT5G22370) involved in embryo development, and the cation calcium exchanger CAX9 (cation calcium exchanger 9; AT3G14070).

These results show that HRS1 possesses two distinct target CREs found in HRS1-activated genes belonging to developmental processes pathways.

HRS1 is post-transcriptionally controlled by $P$ provision. Since HRS1 is clearly strongly transcriptionally activated by the $\mathrm{NO}_{3}^{-}$ signalling pathway (Fig. 1a), and it is involved in the convergence of $\mathrm{Pi}$ and $\mathrm{NO}_{3}^{-}$signals (Fig. 2), we investigated how $\mathrm{Pi}$ and $\mathrm{NO}_{3}^{-}$ might affect the levels of HRS1 protein. As expected for such a $\mathrm{NO}_{3}^{-}$-induced transcript (Fig. 1), the HRS1 protein strongly accumulates in response to $\mathrm{NO}_{3}^{-}$treatment (Fig. 4a). Interestingly, in whole roots, HRS1:GFP protein accumulation was reduced after 24 and $48 \mathrm{~h}$ of $\mathrm{Pi}$ deprivation (Fig. $4 \mathrm{~b}$ ). In the same conditions (48-h Pi starvation), HRS1 mRNA levels are not changed, even if the plant felt the Pi deprivation (as reported by IPS1, Fig. 4b). These findings suggest a possible post-transcriptional regulation of $\mathrm{HRS} 1$ by $\mathrm{Pi}$ provision. Interestingly, this interaction of $\mathrm{NO}_{3}^{-}$transcriptional induction of HRS1, together with its post-transcriptional regulation in response to $-\mathrm{P}$, could constitute a mechanism that entangles both nutritional signals. To confirm this hypothesis, the $\mathrm{NO}_{3}^{-}$transcriptional regulation has to be conserved independent of the preceding $\mathrm{P}$ treatment. To demonstrate this, we verified that the transcriptional regulation of HRS1 by $\mathrm{NO}_{3}^{-}$is maintained in $\mathrm{P}$ varying conditions (Fig. 4c). Furthermore, we observed that the accumulation of the HRS1 protein accumulation is not affected by $\mathrm{P}$ provision in the short-term experiments. This demonstrates that $\mathrm{NO}_{3}^{-}$and $-\mathrm{P}$ signals act early (within minutes) and late (within days) at the transcriptional and post-transcriptional levels, respectively (Fig. 4). To delve further into one potential mechanism of the $\mathrm{P}$ effect on HRS1 activity, we analysed HRS1-GFP protein half-life in $\mathrm{P}$ varying conditions (Fig. 5a,b). We recorded that $-\mathrm{P}$ treatment shifts the HRS1 half-life from $\sim 20$ to $30 \mathrm{~min}$. Interestingly, this mechanism is not restricted to HRS1, since another nitrateregulated protein is under this kind of regulation (Fig. 5c). Indeed, we have found that the nitrate transporter/sensor NRT1.1 is strongly destabilized on $-\mathrm{P}$ conditions (as compared with another membrane protein PIP2.1, Fig. 5c). This demonstrates that $\mathrm{P}$ deficiency conditions may broadly affect nitrate-regulated proteins to convey a layer of the $\mathrm{NO}_{3}^{-}$and $\mathrm{PO}_{4}^{3-}$ signal interaction.

Genome-wide effect of HRS1 and HHO1 on gene expression. To better document the in planta effects from the modification of the HRS1/HHO1 TFs, plantlets (Col, hrs1; hho1, HRS1-OE and HHO1-OE) were grown in $-\mathrm{P} /+\mathrm{NO}_{3}^{-}$conditions for 6 days in three independent experiments. Whole roots (Fig. 6a) were harvested to measure the effect of these HRS1/HHO1 genetic modifications using last generation Affymetrix chips (Arabidopsis
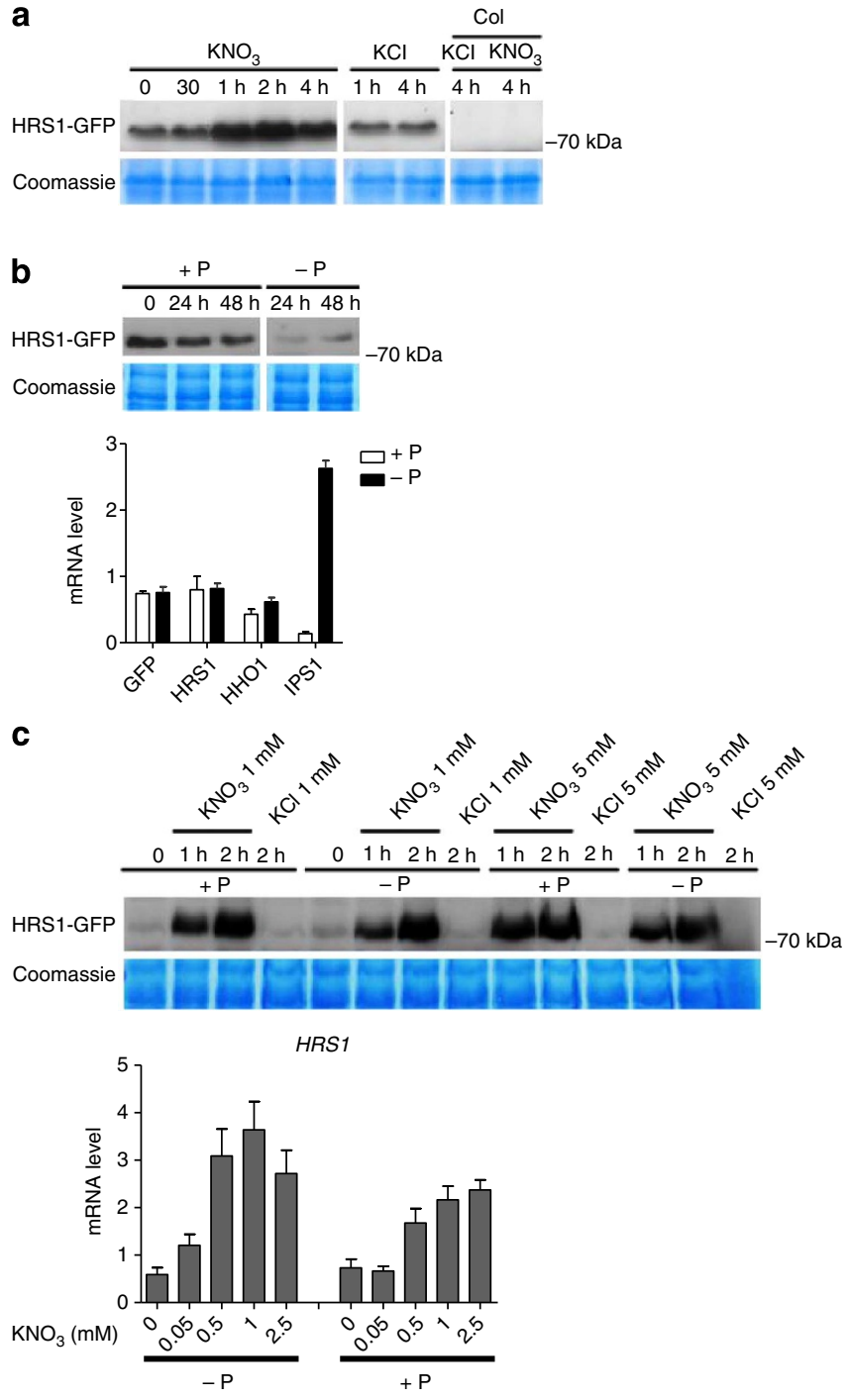

Figure 4 | $\mathrm{P}$ and $\mathrm{NO}_{3}^{-}$provisions influence HRS1 protein accumulation, but the $P$ signal does not affect its mRNA accumulation and $\mathrm{NO}_{3}^{-}$ response. (a) HRS1-GFP protein accumulation is induced in response to $1 \mathrm{mM} \mathrm{NO}_{3}^{-}$treatment. (b) HRS1-GFP protein accumulation is affected by $\mathrm{P}$ provision; $48 \mathrm{~h} \mathrm{P}$ starvation (reported by IPS1 sentinel regulation) does not affect HRS1 (endogenous gene), HHO1, and HRS1:GFP mRNA accumulation. (c) Rapid $\mathrm{NO}_{3}^{-}$transcriptional activation of HRS1 and subsequent protein accumulation is maintained regardless $\mathrm{P}$ provision. Different $\mathrm{NO}_{3}^{-}$levels induce: (i) HRS1-GFP protein accumulation in roots of 48-h P starved plants and (ii) HRS1 transcript accumulation in plant roots grown (14 days) on $\mathrm{P}$ varying media. Values are means \pm s.e.m. $(n=3)$.

gene1.1ST array, Supplementary Data 2). Analysis was performed as previously described in refs 43,44 (for details see Methods). Analysis of variance (ANOVA) followed by a post hoc Tukey test yielded 1,125 HRS1/HHO1-regulated probes corresponding to 969 unambiguous genes. A clustering analysis helped to understand the dominant mode of regulation triggered by the mutation or the overexpression of HRS1 and HHO1 (Fig. 6b). Among these 969 HRS1/HHO1-regulated genes, 22 genes have been identified to be direct HRS1 targets based on the TARGET approach (11 upregulated and 11 downregulated, Supplementary Data 1). This relatively low number is however higher than one would expect by chance (Monte Carlo test $P$ value $<0.05$ ). Among these HRS1 direct targets is NRT1.1, which is found in cluster \#8. Since we have shown that NRT1.1 is a key regulator of 
a

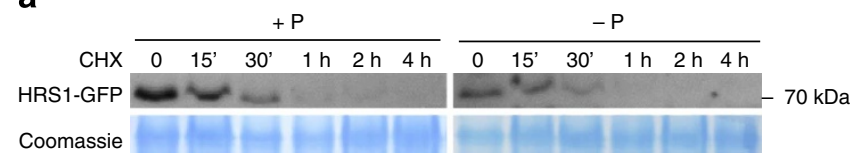

b

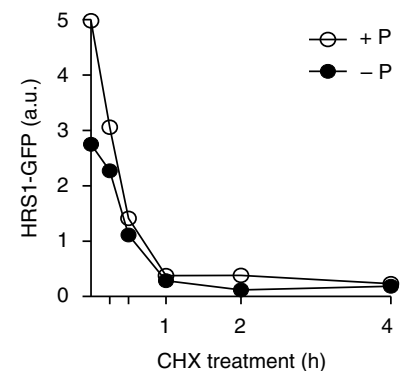

c

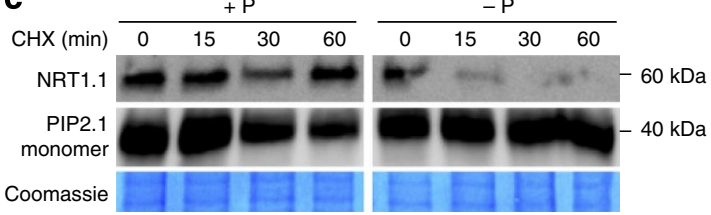

Figure 5 | P provision affects HRS1 and NRT1.1 protein stability. (a) $\mathrm{CHX}$ $(100 \mu \mathrm{M})$ treatment affects HRS1-GFP accumulation differentially in $+\mathrm{P}$ and $-\mathrm{P}$ conditions. (b) Quantification of immunoblot signal. (c) P starvation accelerates the protein degradation rate of the nitrate transporter/sensor NRT1.1, but not the aquaporin PIP2.1 (used as non-nitrate-inducible control).

HRS1 primary $\mathrm{NO}_{3}^{-}$induction (Fig. 1e), this demonstrates a potential feedback mechanism of HRS1 on its own regulator, as is often found in regulatory networks. The 969 HRS1/HHO1regulated genes have been subjected to GO enrichment analysis by the agriGO web site ${ }^{39}$ followed by a REVIGO analysis to reduce and summarize the number of GO terms detected ${ }^{45}$. Interestingly, this analysis demonstrates that 'phosphorus metabolism', 'hormone metabolism', 'starch metabolism', 'developmental process', 'cellular nitrogen compound metabolism', 'post-embryonic development', 'response to red light' and 'response to abiotic stimulus' are (among others; Supplementary Fig. 6) enriched in this list. This demonstrates that (i) HRS1/HHO1 indeed affect the processes that have been predicted by the cellbased TARGET system approach (Fig. 1g; Supplementary Fig. 6), and (ii) that HRS1/HHO1 may have other functions, as the one described herein, some of which that was predicted by other studies (refs 35,46 and discussed below).

To obtain further insights into the altered gene responses triggered by altered expression of HRS1/HHO1, a clustering analysis uncovered 19 clusters of mis-regulated genes (Supplementary Data 2). The overall analysis of the 19 clusters demonstrates that even if HRS1 and HHO1 seem to play redundant roles in the control of primary root growth (Fig. 2), they may control at the same time the same set of genes (such as in cluster $\# 9,17,7,14,10,8$ ) or different genes (such as in clusters $\# 4,11,12,15,13,16)$. AgriGO was then used to determine the molecular coherence of these HRS1/HHO1-regulated gene clusters into potential biomodules (that is, co-regulated genes having a particular function ${ }^{47}$ ). Interestingly, in the clusters for which HRS1 and HHO1 have a coherent effect on gene regulation (control the same set of genes), the GO terms are related to 'stress responsiveness' and 'response to reactive oxygen species (ROS)'. Moreover, it seems that HRS1 overexpression is the one that triggers the most regulation on genes responsible for growth control (that is, cluster \#11). Taken together, this could suggest that HRS1 and HHO1 play redundant functions due to their phylogenetic proximity, but might control different set of genes depending on the genomic context. However, since the two TFs converge towards the control of ROS signalling-related genes, and that ROS have recently been shown to be involved in the control of primary root meristematic activity ${ }^{48}$ or cell size ${ }^{49}$, it is tempting to propose that such a mechanism could be downstream of HRS1/HHO1 influence. It is however important to note that UPB1 does not belong to the $969 \mathrm{HRS} 1 / \mathrm{HHO} 1$-regulated genes based on whole-root transcriptome studies. These hypotheses will need further experiments to be fully validated.

One hypothesis related to the modularity of the HRS1 versus HHO1 effect is that they can affect different genes according to the possible tissue context. To document this possibility, the different HRS1/HHO1-controlled gene clusters were overlapped to a set of genes defined as markers of different root cell layers ${ }^{50}$. Interestingly, clusters \#11,7,17,14,10,8,18 appear to significantly $\left(P\right.$ value $<0.05$, Monte Carlo test named GeneSect ${ }^{22}$ ) overlap with cell type-specific markers (third column, Fig. 6b). Thus, since the transcriptome is performed at the whole-root level, this overlap may show that some clustering properties are related to different activities of the two TFs in different cell types. Again, this will need to be fully validated by following further experimental studies.

The most striking cluster of this analysis is \#8. Indeed, it gathers genes being more highly expressed in the double hrs $1-1$; hho1-1 mutant and repressed by HRS1 or HHO1 overexpression (Fig. 6b). Interestingly, this cluster comprises genes known to be responsive to $\mathrm{NO}_{3}^{-}$(including NRT1.1 and NRT2.4, among others) and hormones. Indeed, 'response to auxin stimulus' is also an over-represented GO term in this gene cluster. This observation opens perspectives concerning the role of auxin signalling in the control of root developmental mechanisms that could explain the effect of HRS1 and HHO1 in - P conditions.

To consolidate the fact that HRS1 binds cis-elements of six genes that are found to be direct targets of HRS1, which are activated in the cell-based system (Figs 1 and 3), we wondered why these genes are not found in the 969 HRS1-regulated genes detected in whole roots? Since the P effect is very much localized to the root apex, and likely not affected by the whole-plant $\mathrm{P}$ status (according to transfer experiments Supplementary Fig. 3), we hypothesized that the regulation by P may be restricted to the most apical part of the root. To validate this hypothesis, we performed transfer experiments from $+\mathrm{P} /+\mathrm{N}$ to $-\mathrm{P} /+\mathrm{N}$ media. The tip of the primary root $(2-3 \mathrm{~mm})$ was harvested from the WT, hrs1-1;hho1-1 double mutant and overexpressors and RNA used for qPCR experiments. This revealed that RAP2.7, NF-YB5 and the DEAD helicase are indeed regulated by HRS1 overexpression at the root apex (Fig. 6c). Several meristem-related genes (CLV1, FAS1 and TSO1), defined as direct targets by the cell-based TARGET system (Supplementary Data 1), were also measured and displayed misregulation in the hrs1;hhol double mutant, but with no effect of the TF overexpression (Fig. 6c). This demonstrates that these genes may be indeed under the direct influence of HRS1 in plants, and are potential candidates controlling meristematic activity together with the previously evoked pathways that include auxin and ROS signalling (refers to clusters \#8 and \#9,15,11, respectively, Fig. 6 b). To decipher the different regulatory pathways below the action of the genes known to sensitize primary root to local $\mathrm{P}$ deficiency, it will be important to confront whole-genome expression reprogramming induced by $l p r 1 / 2$ (ref. 8), pdr2 (ref. 9) and hrs1;hhol to define whether these regulatory modules share common signalling pathways. Further, reverse genetic studies will also be needed to validate that the potential regulators of meristematic activity such as CLV1, FAS1, TSO1, RAP2.7 or NF-YB5 are indeed a part of the HRS1/HHO1-mediated response. 
a

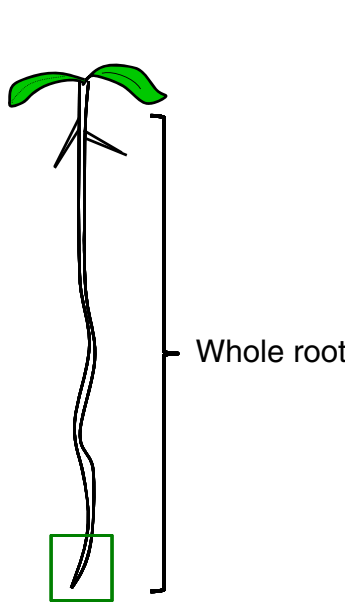

2-3 mm apex
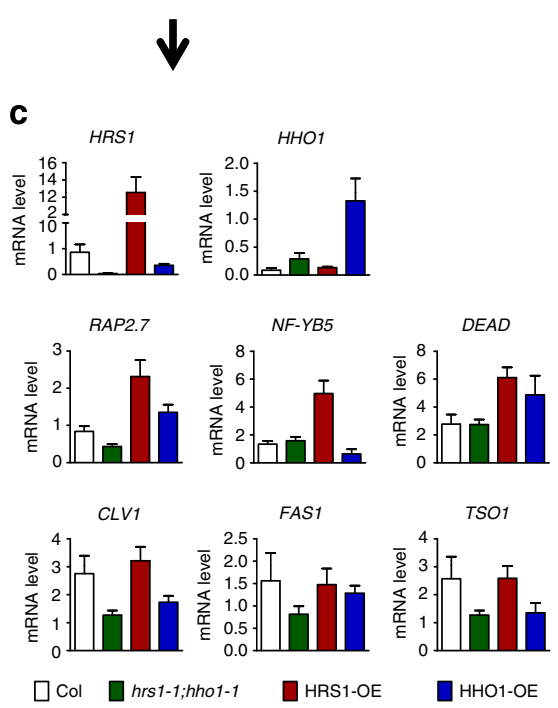

TSO1

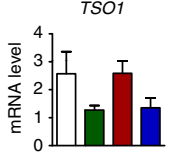

HHO1-OE b
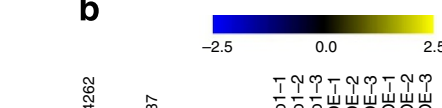

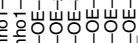

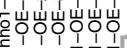

$\begin{array}{lll}2 & 0\end{array}$

\begin{tabular}{|l|l|l|}
\hline \multicolumn{1}{|c|}{ Over-represented GO terms ( $P$ value) } & $\begin{array}{l}\text { Remarkable } \\
\text { genes }\end{array}$ & $\begin{array}{l}\text { Significant } \\
\text { overlap }\end{array}$ \\
\hline GO:0009791 post-embryonic development 0.0078 & $\begin{array}{l}\text { AGL12 AGL14 } \\
\text { AGL22 PID } \\
\text { GO:0019825 oxygen binding 0.00044 }\end{array}$ & \\
GO:0048869 cellular developmental process 0.009 & $\begin{array}{l}\text { CYCB2;1 } \\
\text { Pectin-lyase }\end{array}$ & Phloem \\
\hline
\end{tabular}

GO:0044262 cellular carbohydrate metabolic process 0.0014

GO:0034641 cellular etc nitrogen compound metabolic

GO:0016491 oxidoreductase activity 0.0073

GO:0009408 response to heat $2.8 \mathrm{e}-05$

GO:0006950 response to stress 0.00036

GO:0000302 response to reactive oxygen species 0.00088

P

\begin{tabular}{|lll|l|}
\hline & RAP2.4 & \\
\hline 17 & PING & \\
\hline GO:0006950 response to stress 0.00036 & \\
\hline
\end{tabular}

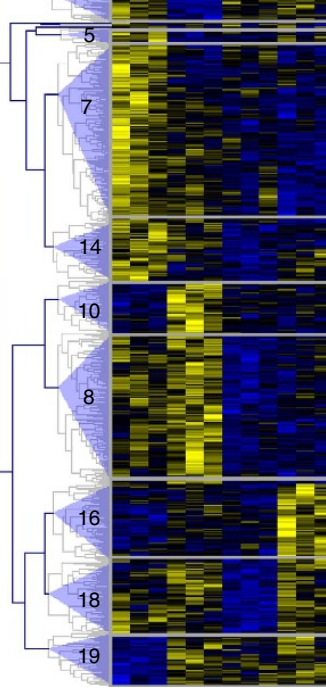

GO:0010200 response to chitin 0.00094

GO:0010114 response to red light $1.4 \mathrm{e}-05$

GO:0034641 cellular etc nitrogen compound metabolic

process 0.00071

GO:0012505 endomembrane system 0.0084

GO:0010017 red or far-red light signalling pathway 2.2e-05

GO:0007242 intracellular signalling cascade 0.047

GO:0001883 purine nucleoside binding 0.014

GO:0010114 response to red light 0.002

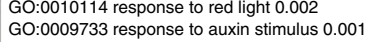

GO:0006807 nitrogen compound metabolic process 0.0077

GO:0015075 ion transmembrane transporter activity 0.00024

GO:0006950 response to stress 0.014

GO:0043412 macromolecule modification 0.048

GO:0006790 sulfur metabolic process 0.00042

GO:0005773 vacuole 0.023

GO:0033036 macromolecule localization 0.015

GO:0009507 chloroplast 0.03

Cluster\#

Figure 6 | Probing the effect of HRS1 and HHO1 mutations and overexpressions in - P-deficient root transcriptome. (a) Six-day-old plantlets were grown on $-\mathrm{P} /+\mathrm{NO}_{3}^{-}$conditions (b) or on $+\mathrm{P} /+\mathrm{NO}_{3}^{-}$media and then transferred 2 days to $-\mathrm{P} /+\mathrm{NO}_{3}^{-}$conditions (c). (b) Clustering of the 969 HRS1-regulated genes from whole roots of plantlets grown on $-\mathrm{P} /+\mathrm{NO}_{3}^{-}$media. For each cluster, a selection of over-represented $\mathrm{GO}$ terms is given, as well as remarkable genes belonging to the cluster. Clusters overlap with $\mathrm{NO}_{3}^{-}$(ref. 31); hormonal ${ }^{59}$ responsive genes or root cell type-specific markers ${ }^{50}$ have been measured using the GeneSect algorithm ${ }^{22}$. Significant overlaps $(P$ value $<0.05)$ are reported in the third column. $(\mathbf{c})$ Reverse transcription qPCR analysis on selected HRS1 direct targets on RNA extracted from 2- to 3-mm sections of the root apex in a transfer experiment. Values are means \pm s.e.m. $(n=3)$. Residual $\mathrm{HHO} 1$ expression in the mutant is explained in Supplementary Fig. 2.

Finally, we believe that this transcriptomic analysis provides an important proxy towards understanding the other roles of HRS1 in plants. Indeed, it has been shown to be controlling germination ${ }^{51}$, or to confer drought or salt stress tolerance ${ }^{52}$. Again the general functions regulated by HRS1 such as 'ROS signalling', as well the important overlap with $\mathrm{ABA}$ responsive genes are important keys to understand the underlying mechanisms behind the previously reported phenotypes.

\section{Discussion}

As we take together the above observations, we propose the model described in Fig. 7. HRS1 is under a dual regulation: (i) a $\mathrm{NO}_{3}^{-}$-induced (NRT1.1/CHL1 NLP7 dependent) transcriptional control, and (ii) a Pi-regulated post-transcriptional control. In response to these two signals, HRS1 controls primary root growth through possibly: (i) its action on two independent CREs contained in promoters of activated genes; and (ii) its effect on pathways such as ROS or Auxin signalling. These two possibilities are not exclusive. Our observations can, to some extent, be related to the recent work of Kellermeier et al. ${ }^{53}$, which makes a clear demonstration that nutrient signalling interaction is rather a general rule than an exception.

Our work provides a potential mechanism for understanding how the two key plant mineral nutrients $\left(\mathrm{PO}_{4}{ }^{3-}\right.$ and $\left.\mathrm{NO}_{3}{ }^{-}\right)$ interact to control root growth, an issue of global importance, since nitrogen and phosphorus are the two most widely used fertilizers worldwide that maintain plant growth and production. For instance, a recent work by Delgado-Baquerizo et al. showed that at the ecological level, drying lands might impact markedly nitrogen and phosphorus ratios available for plants, thus jeopardizing food production worldwide ${ }^{54}$. The molecular events, mediated by HRS1 and HHO1 in plants, that integrate two mineral-related signalling pathways are part of the fundamental knowledge needed to tackle such key environmental challenge in a global warming context. 


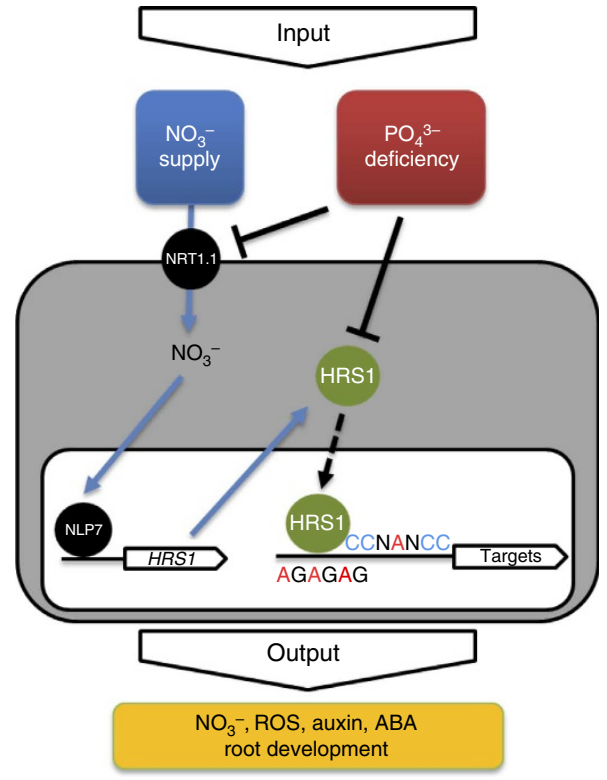

Figure 7 | Proposed model: $\mathrm{NO}_{3}^{-}$and $\mathrm{P}$ limitation signals are integrated by the control of HRS1 at the transcriptional and post-transcriptional level, respectively. On $\mathrm{NO}_{3}^{-}$treatment, the NRT1.1 and NLP7 regulatory module induces rapid HRS1 transcript and protein accumulation. The prolonged $\mathrm{P}$ deficiency condition negatively affects the accumulation of HRS1 and modifies its regulatory activity. Furthermore, the P limitation signal seems to control the $\mathrm{NO}_{3}^{-}$sensor (NRT1.1) protein half-life. This delineates a working model to understand several points of $\mathrm{NO}_{3}^{-}$and $\mathrm{PO}_{4}^{3-}$ signal interactions via the HRS1 pathway.

\section{Methods}

Plant material. All $A$. thaliana plants were in the Columbia background. Mutant hrs1-1 (SALK_067074), hho1-1 (SAIL_28_D03) and mutant chl1-12 (Salk_034596) were obtained from ABRC seeds stock center. The hrs1-1;hho1-1 double mutant has been obtained by crossing. Despite the presence of a highly accumulated chimeric RNA in the hho1-1 line (characterized in Supplementary Fig. 2c), the production of a full-length gene product is knocked out (Supplementary Fig. 2b). The promoter gene GFP lines for tissue localization were obtained by cloning HRS1 and $\mathrm{HHO}$ from genomic sequences, bringing respectively the $3-$ and $2.5-\mathrm{kb}$ upstream promoter region and the gene, into pMDC107 Gateway-compatible vector. Overexpressor lines were obtained by cloning HRS1- and HHO1-coding sequences into pMDC32 Gateway-compatible vector ${ }^{55}$, using primers listed in Supplementary Data 1. The constructs were transferred to Agrobacterium tumefaciens strain GV3101 and used for the Arabidopsis transformation by the floral dip method ${ }^{56}$. Transgenic plants were selected by antibiotic resistance and T3 homozygote descendants were used for analysis.

Growth conditions and treatments. For $\mathrm{NO}_{3}^{-}$treatment experiment, plants were grown in sterile hydroponic conditions as described in ref. 22. Hydroponic media consisted of MS basal salt medium containing no nitrate and supplemented with $3 \mathrm{mM}$ sucrose, $0.5 \mathrm{mM}$ ammonium succinate, MES buffered at $\mathrm{pH} 5.7\left(0.5 \mathrm{gl}^{-1}\right)$. Plants were grown for 15 days in day/night cycles $(16 / 8 \mathrm{~h} ; 65 \mu \mathrm{mol}$ photons $\mathrm{m}^{-2} \mathrm{~s}^{-1}$ ) at $22^{\circ} \mathrm{C}$. Plants were transferred to an equivalent fresh nitrogen-free medium for $24 \mathrm{~h}$ and then treated with nitrogen as $1 \mathrm{mM} \mathrm{KNO}$ or $1 \mathrm{mM} \mathrm{KCl}$, as mock treatment. Roots were sampled at different time points after the treatment and immediately frozen in liquid nitrogen. For P starvation experiments, Col-0 and hrs 1-1; hho1-1 seeds were sown on the surface of solid media consisting of MS basal salt medium nitrogen and phosphorous-free supplemented with $\mathrm{KNO}_{3}$ at different concentrations $(0.05,0.5,1$ and $2.5 \mathrm{mM}), 0.5 \mathrm{mM} \mathrm{KH}_{2} \mathrm{PO}_{4}$ for P-sufficient condition, $3 \mathrm{mM}$ sucrose, MES $\left(0.5 \mathrm{gl}^{-1}\right)$ and $0.8 \%(\mathrm{w} / \mathrm{v})$ agarose. Different volumes of $1 \mathrm{mM} \mathrm{KCl}$ solution were added to the media to keep the $\mathrm{K}^{+}$ concentration constant among different conditions. Plants were grown vertically for 9 days in day/night cycles $\left(16 / 8 \mathrm{~h} ; 90 \mu \mathrm{mol}\right.$ photons $\left.\mathrm{m}^{-2} \mathrm{~s}^{-1}\right)$ at $22^{\circ} \mathrm{C}$.

For P starvation experiments in liquid media, plants were grown for 15 days in sterile hydroponics conditions, in MS media containing $5 \mathrm{mM} \mathrm{KNO}_{3}$ and $1 \mathrm{mM}$ $\mathrm{KH}_{2} \mathrm{PO}_{4}$. Plants were transferred towards an equivalent $\mathrm{KH}_{2} \mathrm{PO}_{4}$-free fresh medium $(-\mathrm{P})$ or $1 \mathrm{mM} \mathrm{KH}_{2} \mathrm{PO}_{4}$ media $(+\mathrm{P})$ for $48 \mathrm{~h}$. For half-life experiment, CHX was used at $100 \mu \mathrm{M}$. All growth and gene expression experiments are supported by at least three independent experiments.
Root growth measurements. Beginning 2 days after seed sowing, two Petri dishes per condition (that is, at least 30 plants) were scanned every day at 400 d.p.i. Root length was measured using Optimas 6.1 software and statistical differences between genotypes were calculated using Student's $t$-test.

Real-time qPCR analysis. Total RNA was isolated from Arabidopsis roots and shoots using TriReagent (Molecular Research center Inc.) and digested with DNAseI (Sigma-Aldrich, St Louis, USA). Total RNAs were then reverse transcribed to one-strand complementary DNA using Thermo script RT (Invitrogen) according to the manufacturer's protocol. Gene expression was determined by reverse transcription qPCR (LightCycler 480; Roche Diagnostics, Basel, Switzerland) using gene-specific primers (listed in Supplementary Data 1) and LightCycler 480 SYBR Green I Master mix (Roche, IN, USA). Expression levels of tested genes were normalized to expression levels of the actin and clathrin genes.

TF perturbation assays in the TARGET system. The TARGET procedure has been performed as previously described in Bargmann et al. ${ }^{36}$ Protoplasts were treated with $35 \mu \mathrm{M}$ CHX and $10 \mu \mathrm{M}$ DEX. The red fluorescent protein was used as marker selection for fluorescent-activated cell sorting of successfully transformed protoplasts. One milimolar $\mathrm{NO}_{3}^{-}$was maintained during the whole procedure. RNA was extracted and amplified for hybridization with ATH1 Affymetrix chips. Data were analysed with R. Mas5 normalized data were extracted and analysed by a $t$-test procedure for DEX response $(P$ value $<0.05$, fold regulation $>2 \times$, corresponding to a false discovery rate $<0.1$.

Microscopy. Col-0, pHRS1:HRS1:GFP and pHHO1:HHO1:GFP seedlings were grown on half-strength MS media for 8 days. The same liquid media was used for mounting plantlets during the image capture. Fluorescence imaging was performed using the Olympus BX61 microscope. Samples were excited at $470 \mathrm{~nm}$ and emission was collected between 500 and $535 \mathrm{~nm}$. Confocal images were performed with a Zeiss LSM510 Meta laser scanning microscope. Root cell walls were stained with propidium iodine $10 \mu \mathrm{g} \mathrm{ml}^{-1}$ (Sigma) and nucleus with 4,6-diamidino-2phenylindole $1 \mu \mathrm{g} \mathrm{ml}^{-1}$ (Sigma). Three-dimensional root reconstructions were obtained from confocal $\mathrm{Z}$ series using the Imaris software.

Semantic gene enrichment analysis. To identify the most represented gene functions for each gene list, the gene descriptions of targets were retrieved from TAIR web site (http://www.arabidopsis.org/index.jsp) and the list was analysed using a software developed in R (http://www.r-project.org/). Briefly, given the list of 551 HRS1 up-/downregulated genes, the software counts the occurrence of each term of the description and compares it with the occurrence of the same term in 1,000 random lists of the same size. Results are presented as a cloud of words, whose colour and size are correlated to their occurrence.

Phylogenetic analysis. The phylogeny reconstruction was inferred by using the maximum likelihood method. The 40 sequences coding for G2-like proteins were retrieved from the AGRIS (Arabidopsis Gene Regulatory Information Server; http://arabidopsis.med.ohio-state.edu/) database. The bootstrap values were obtained based on 500 replicates. Evolutionary analysis was conducted in MEGA5 software ${ }^{57}$.

Expression and purification of recombinant GST-HRS1 protein. HRS1 coding sequence CDS was first cloned in pDONR207, using the primers listed in Supplementary Data 1 and then transferred to pDEST15 vector (Invitrogen) by LR reaction following the manufacturer's instructions. The GST-HRS1 fusion protein was expressed in Escherichia coli Rosetta 2(DE3)pLysS (Novagen, Darmstadt, Germany) cells after induction with $1 \mathrm{mM}$ isopropyl- $\beta$-D-thiogalactoside for $16 \mathrm{~h}$ at $22^{\circ} \mathrm{C}$. Bacteria were harvested, suspended in PBS buffer containing lysozyme from chicken egg white (Sigma) and complete protease inhibitor cocktail (Roche) and sonicated. The protein extract was purified on glutathione sepharose beads (GE Healthcare, Freiburg, Germany), eluted with $10 \mathrm{mM}$ reduced glutathione (Sigma), $50 \mathrm{mM}$ Tris buffer and dialysed overnight in $150 \mathrm{mM} \mathrm{NaCl}, 50 \mathrm{mM}$ HEPES, pH 7.4 buffer.

EMSA. Purified GST-HRS1 recombinant protein was used to determine DNA binding by EMSA. Single-stranded DNA oligonucleotides (listed in Supplementary Data 1) were biotin labelled using the Biotin $3^{\prime}$ End DNA Labelling Kit (Thermo Scientific) and complementary pairs were annealed to make double-stranded DNA probes. The binding of recombinant protein $(50 \mathrm{ng})$ to the biotin-labelled probes (20 fmol) was carried out in a reaction mixture containing $10 \mathrm{mM}$ Tris, $50 \mathrm{mM}$ $\mathrm{KCl}, 1 \mathrm{mM}$ DTT, pH 7.5, 2.5\% glycerol, $5 \mathrm{mM} \mathrm{MgCl}_{2}, 1 \mu \mathrm{g}$ poly (deoxyinosinicdeoxycytidylic) and $0.05 \%$ NP- 40 . After incubation at $22^{\circ} \mathrm{C}$ for $30 \mathrm{~min}$, the protein-probe mixture was separated in a $4 \%$ polyacrylamide native gel and transferred to a Biodyne B Nylon membrane by capillary action in $20 \times$ SSC buffer overnight (Thermo Scientific). After ultraviolet crosslinking $(254 \mathrm{~nm})$ for $90 \mathrm{~s}$ at $120 \mathrm{~mJ} \cdot \mathrm{cm}^{-2}$, the migration of biotin-labelled probes was detected using streptavidin horseradish peroxidase conjugates in the Chemioluminescent Nucleic Acid 
Detection Module (Thermo Scientific) and exposed to X-ray film. As a negative control, we tested that the GST tag has no affinity for HRS1-related CREs (Supplementary Fig. 5).

Protein extraction and immunoblot analysis. Roots were sampled and frozen in liquid nitrogen. Total proteins were obtained using the Plant Total Protein Extraction Kit (Sigma) and quantified with the Pierce $660 \mathrm{~nm}$ Protein Assay using a BSA standard curve. Membrane proteins were obtained by disrupting roots in a buffer containing a plant anti-protease cocktail (Sigma-Aldrich) and antiphosphatases (30 mM glycerophosphate, $5 \mathrm{mM}$ molybdate and $10 \mathrm{mM} \mathrm{NaF}$ ). The whole-membrane fraction was then isolated by centrifugation $(100,000 \mathrm{~g}, 4 \mathrm{~h})$ on a $55 \%$ sucrose cushion. Immunoblot analysis was performed on $40-50 \mu \mathrm{g}$ of proteins using anti-GFP-HRP 1:2,500 (Miltenyi Biotec, 130-091-833), anti-NRT1.1 1:5,000 (AS12 2611, Agrisera) and anti-PIP2.1 1:5,000 (ref. 58). Coomassie Brilliant Blue staining of blots was used to control the protein levels after electrotransfer. Uncropped versions of the blots are provided in Supplementary Figs 7 and 8. Band intensity quantification was performed using a chemioluminiescent image analyser LAS3000 (Fujifilm) and ImageGauge (Fujifilm) software.

Transcriptome analysis. The transcriptome analysis was performed using the GeneChip Whole Transcript (WT) Expression Array following the manufacturer's protocol. Total RNA was isolated from 6-day-old roots grown on media containing $2.5 \mathrm{mM} \mathrm{KNO}_{3}$ and no $\mathrm{PO}_{4}^{3-}$ (three independent experiments). RNA integrity numbers were checked by microfluidic analysis in an Agilent 2100 Bioanalyzer. Complementary DNAs were prepared from $150 \mathrm{ng}$ of total RNA following the WT Plus Reagent Kit protocol, hybridized on Affymetrix Array Strip and processed on the GeneAtlas System. Data set analysis was performed with R. A one-way ANOVA model was applied (four levels: Col, hrs1-1;hho1-1, HRS1-OE and HHO1-OE) followed by a post hoc Tukey test (R functions aov() and TukeyHSD()). Any probe having a significant $P$ value $<0.05$ for the ANOVA or the Tukey test was kept for further analysis. Clustering analysis was performed with $\mathrm{MeV}$ software (distance, Pearson correlation).

\section{References}

1. Gruber, B. D., Giehl, R. F., Friedel, S. \& von Wiren, N. Plasticity of the Arabidopsis root system under nutrient deficiencies. Plant Physiol. 163, 161-179 (2013).

2. Peret, B., Clement, M., Nussaume, L. \& Desnos, T. Root developmental adaptation to phosphate starvation: better safe than sorry. Trends Plant Sci. 16, 442-450 (2011).

3. Alvarez, J. M., Vidal, E. A. \& Gutierrez, R. A. Integration of local and systemic signaling pathways for plant $\mathrm{N}$ responses. Curr. Opin. Plant Biol. 15, 185-191 (2012).

4. Vidal, E. A. \& Gutierrez, R. A. A systems view of nitrogen nutrient and metabolite responses in Arabidopsis. Curr. Opin. Plant Biol. 11, 521-529 (2008)

5. Gojon, A., Nacry, P. \& Davidian, J. C. Root uptake regulation: a central process for NPS homeostasis in plants. Curr. Opin. Plant Biol. 12, 328-338 (2009).

6. Krouk, G., Crawford, N. M., Coruzzi, G. M. \& Tsay, Y. F. Nitrate signaling: adaptation to fluctuating environments. Curr. Opin. Plant Biol. 13, 266-273 (2010).

7. Camacho-Cristobal, J. J. et al. PRD, an Arabidopsis AINTEGUMENTA-like gene, is involved in root architectural changes in response to phosphate starvation. Planta 228, 511-522 (2008).

8. Svistoonoff, S. et al. Root tip contact with low-phosphate media reprograms plant root architecture. Nature Genet. 39, 792-796 (2007).

9. Ticconi, C. A. et al. ER-resident proteins PDR2 and LPR1 mediate the developmental response of root meristems to phosphate availability. Proc. Natl Acad. Sci. USA 106, 14174-14179 (2009).

10. Ticconi, C. A., Delatorre, C. A., Lahner, B., Salt, D. E. \& Abel, S. Arabidopsis pdr2 reveals a phosphate-sensitive checkpoint in root development. Plant J. 37, 801-814 (2004)

11. Rubio, V. et al. A conserved MYB transcription factor involved in phosphate starvation signaling both in vascular plants and in unicellular algae. Genes Dev. 15, 2122-2133 (2001).

12. Bustos, R. et al. A central regulatory system largely controls transcriptional activation and repression responses to phosphate starvation in Arabidopsis. PLoS Genet. 6, el001102 (2010).

13. Miura, K. et al. The Arabidopsis SUMO E3 ligase SIZ1 controls phosphate deficiency responses. Proc. Natl Acad. Sci. USA 102, 7760-7765 (2005).

14. Vidal, E. A., Tamayo, K. P. \& Gutierrez, R. A. Gene networks for nitrogen sensing, signaling, and response in Arabidopsis thaliana. Wiley Interdiscip. Rev. Syst. Biol. Med. 2, 683-693 (2010).

15. Walch-Liu, P. \& Forde, B. G. Nitrate signalling mediated by the NRT1.1 nitrate transporter antagonises L-glutamate-induced changes in root architecture. Plant J. 54, 820-828 (2008).
16. Kant, S., Peng, M. \& Rothstein, S. J. Genetic regulation by NLA and microRNA827 for maintaining nitrate-dependent phosphate homeostasis in arabidopsis. PLoS Genet. 7, e1002021 (2011).

17. Lin, W. Y., Huang, T. K. \& Chiou, T. J. Nitrogen limitation adaptation, a target of microRNA827, mediates degradation of plasma membrane-localized phosphate transporters to maintain phosphate homeostasis in Arabidopsis. Plant Cell 26, 454-464 (2013).

18. Peng, M. et al. Adaptation of Arabidopsis to nitrogen limitation involves induction of anthocyanin synthesis which is controlled by the NLA gene. J. Exp. Bot. 59, 2933-2944 (2008).

19. Khamis, S., Lamaze, T., Lemoine, Y. \& Foyer, C. Adaptation of the photosynthetic apparatus in maize leaves as a result of nitrogen limitation: relationships between electron transport and carbon assimilation. Plant Physiol. 94, 1436-1443 (1990).

20. Krouk, G. et al. Nitrate-regulated auxin transport by NRT1.1 defines a mechanism for nutrient sensing in plants. Dev. Cell 18, 927-937 (2010).

21. Ho, C. H., Lin, S. H., Hu, H. C. \& Tsay, Y. F. CHL1 functions as a nitrate sensor in plants. Cell 138, 1184-1194 (2009).

22. Krouk, G., Mirowski, P., LeCun, Y., Shasha, D. E. \& Coruzzi, G. M. Predictive network modeling of the high-resolution dynamic plant transcriptome in response to nitrate. Genome Biol. 11, R123 (2010).

23. Scheible, W. R. et al. Genome-wide reprogramming of primary and secondary metabolism, protein synthesis, cellular growth processes, and the regulatory infrastructure of Arabidopsis in response to nitrogen. Plant Physiol. 136, 2483-2499 (2004).

24. Wang, R. et al. Genomic analysis of the nitrate response using a nitrate reductase-null mutant of Arabidopsis. Plant Physiol. 136, 2512-2522 (2004)

25. Hu, H. C., Wang, Y. Y. \& Tsay, Y. F. AtCIPK8, a CBL-interacting protein kinase, regulates the low-affinity phase of the primary nitrate response. Plant $J$ 57, 264-278 (2009)

26. Sawaki, N. et al. A nitrate-inducible GARP family gene encodes an autorepressible transcriptional repressor in rice. Plant Cell Physiol. 54, 506-517 (2013).

27. Waters, M. T. et al. GLK transcription factors coordinate expression of the photosynthetic apparatus in Arabidopsis. Plant Cell 21, 1109-1128 (2009).

28. Kerstetter, R. A., Bollman, K., Taylor, R. A., Bomblies, K. \& Poethig, R. S. KANADI regulates organ polarity in Arabidopsis. Nature 411, 706-709 (2001)

29. Liu, H. et al. Overexpressing HRS1 confers hypersensitivity to low phosphateelicited inhibition of primary root growth in Arabidopsis thaliana. J. Integr. Plant Biol. 51, 382-392 (2009).

30. Bolle, C. et al. GABI-DUPLO: a collection of double mutants to overcome genetic redundancy in Arabidopsis thaliana. Plant J. 75, 157-171 (2013).

31. Wang, R. et al. Genomic analysis of the nitrate response using a nitrate reductase-null mutant of Arabidopsis. Plant Physiol. 136, 2512-2522 (2004).

32. Wang, R., Xing, X., Wang, Y., Tran, A. \& Crawford, N. M. A genetic screen for nitrate regulatory mutants captures the nitrate transporter gene NRT1.1. Plant Physiol. 151, 472-478 (2009).

33. Konishi, M. \& Yanagisawa, S. Arabidopsis NIN-like transcription factors have a central role in nitrate signalling. Nat. Commun. 4, 1617 (2013).

34. Marchive, C. et al. Nuclear retention of the transcription factor NLP7 orchestrates the early response to nitrate in plants. Nat. Commun. 4, 1713 (2013).

35. Canales, J., Moyano, T. C., Villarroel, E. \& Gutiérrez, R. A. Systems analysis of transcriptome data provides new hypotheses about Arabidopsis root response to nitrate treatments. Front. Plant Sci. 5, 22 (2014).

36. Bargmann, B. O. et al. TARGET: a transient transformation system for genomewide transcription factor target discovery. Mol. Plant 6, 978-980 (2013).

37. Para, A. et al. Hit-and-run transcriptional control by bZIP1 mediates rapid nutrient signaling in Arabidopsis. Proc. Natl Acad. Sci. USA 111, 10371-10376 (2014).

38. Katari, M. S. et al. VirtualPlant: a software platform to support systems biology research. Plant Physiol. 152, 500-515 (2010).

39. Du, Z., Zhou, X., Ling, Y., Zhang, Z. \& Su, Z. agriGO: a GO analysis toolkit for the agricultural community. Nucleic Acids Res. 38, W64-W70 (2010).

40. Bailey, T. L. et al. MEME Suite: tools for motif discovery and searching. Nucleic Acids Res. 37, W202-W208 (2009).

41. Giraud, E. et al. TCP transcription factors link the regulation of genes encoding mitochondrial proteins with the circadian clock in Arabidopsis thaliana. Plant Cell 22, 3921-3934 (2010).

42. Franco-Zorrilla, J. M. et al. DNA-binding specificities of plant transcription factors and their potential to define target genes. Proc. Natl Acad. Sci. USA 111 2367-2372 (2014).

43. Obertello, M., Krouk, G., Katari, M. S., Runko, S. J. \& Coruzzi, G. M. Modeling the global effect of the basic-leucine zipper transcription factor 1 (bZIP1) on nitrogen and light regulation in Arabidopsis. BMC Sys. Biol. 4, 111 (2010).

44. Krouk, G. et al. A systems approach uncovers restrictions for signal interactions regulating genome-wide responses to nutritional cues in Arabidopsis. PLoS Comput. Biol. 5, e1000326 (2009). 
45. Supek, F., Bosnjak, M., Skunca, N. \& Smuc, T. REVIGO summarizes and visualizes long lists of gene ontology terms. PLoS ONE 6, e21800 (2011).

46. Alvarez, J. M. et al. Systems approach identifies TGA1 and TGA4 transcription factors as important regulatory components of the nitrate response of Arabidopsis thaliana roots. Plant J. 80, 1-13 (2014).

47. Nero, D., Krouk, G., Tranchina, D. \& Coruzzi, G. M. A system biology approach highlights a hormonal enhancer effect on regulation of genes in a nitrate responsive "biomodule". BMC Sys. Biol. 3, 59 (2009).

48. Tsukagoshi, H., Busch, W. \& Benfey, P. N. Transcriptional regulation of ROS controls transition from proliferation to differentiation in the root. Cell 143, 606-616 (2010).

49. Lu, D., Wang, T., Persson, S., Mueller-Roeber, B. \& Schippers, J. H. Transcriptional control of ROS homeostasis by KUODA1 regulates cell expansion during leaf development. Nat. Commun. 5, 3767 (2014).

50. Bargmann, B. O. et al. A map of cell type-specific auxin responses. Mol. Sys Biol. 9, 688 (2013).

51. Wu, C. et al. HRS1 acts as a negative regulator of abscisic acid signaling to promote timely germination of Arabidopsis seeds. PLoS ONE 7, e35764 (2012).

52. Mito, T., Seki, M., Shinozaki, K., Ohme-Takagi, M. \& Matsui, K. Generation of chimeric repressors that confer salt tolerance in Arabidopsis and rice. Plant Biotechnol. J. 9, 736-746 (2011).

53. Kellermeier, F. et al. Analysis of the root system architecture of arabidopsis provides a quantitative readout of crosstalk between nutritional signals. Plant Cell 26, 1480-1496 (2014).

54. Delgado-Baquerizo, M. et al. Decoupling of soil nutrient cycles as a function of aridity in global drylands. Nature 502, 672-676 (2013).

55. Curtis, M. D. \& Grossniklaus, U. A gateway cloning vector set for high-throughput functional analysis of genes in planta. Plant Physiol. 133, 462-469 (2003).

56. Clough, S. J. \& Bent, A. F. Floral dip: a simplified method for Agrobacteriummediated transformation of Arabidopsis thaliana. Plant J. 16, 735-743 (1998).

57. Tamura, K. et al. MEGA5: molecular evolutionary genetics analysis using maximum likelihood, evolutionary distance, and maximum parsimony methods. Mol. Biol. Evol. 28, 2731-2739 (2011).

58. Santoni, V., Vinh, J., Pflieger, D., Sommerer, N. \& Maurel, C. A proteomic study reveals novel insights into the diversity of aquaporin forms expressed in the plasma membrane of plant roots. Biochem J. 373, 289-296 (2003).

59. Nemhauser, J. L., Hong, F. \& Chory, J. Different plant hormones regulate similar processes through largely nonoverlapping transcriptional responses. Cell 126, 467-475 (2006).

\section{Acknowledgements}

We thank Benoit Lacombe and Frederic Gaymard for the comments and discussions; Elodie Jublanc from the Montpellier Rio Imaging (MRI) core facility for the confocal assistance and three-dimensional imaging reconstruction; Tony Sierra for the technical assistance during Gene Atlas analysis; Veronique Santoni for the PIP2.1 antibody; and Franck Lecocq, Rogatien Picaud, Guy Ruiz, Thierry Dessup, and Hugues Baudot for the technical support and plant care. This work was supported by the French Agence Nationale de la Recherche (NitroNet: ANR 11 PDOC 020 01) and Centre National de la Recherche Scientifique (PEPS Bio math Info 2012-2013: SuperRegNet) to G.K. Work on the transcriptional networks was supported NIH R01-GM032877 to G.C. and NIH NRSA-GM095273 to A.M.-C. Results on root architecture responses are supported by NSF MCB-0929338 to G.C. Bioinformatics analysis was supported by the VirtualPlant platform (www.virtualplant.org) developed under NSF DBI-0445666 to G.C. Work by R.W. and N.C. was supported by the National Science Foundation grants (IOS-1021380 and MCB-0929338)

\section{Author contributions}

G.K. designed the project. A.M., A.M.-C., E.R., W.S., R.W., S.R. and G.K. performed the experiments and analysed the data. A.M., A.G., N.M.C., S.R., G.M.C. and G.K. contributed to the study design during the project course. A.M. and G.K. wrote the paper.

\section{Additional information}

Accession codes: Microarray data associated with this study has been deposited in NCBI GEO database under the accession code GSE64352.

Supplementary Information accompanies this paper at http://www.nature.com/ naturecommunications

Competing financial interests: The authors declare no competing financial interests.

Reprints and permission information is available online at http://npg.nature.com/ reprintsandpermissions/

How to cite this article: Medici, A. et al. AtNIGT1/HRS1 integrates nitrate and phosphate signals at the Arabidopsis root tip. Nat. Commun. 6:6274 doi: $10.1038 /$ ncomms7274 (2015). 